\title{
Diffusion Weighted MR Imaging of Breast and Correlation of Prognostic Factors in Breast Cancer
}

\author{
İnci Kızıldağ Yırgın 1 , Gözde Arslan², Enis Öztürk³, Hakan Yırgın", Nihat Taşdemir ${ }^{5}$, \\ Ayşegül Akdoğan Gemici' ${ }^{6}$, Fatma Çelik Kabul ${ }^{3}$, Eyüp Kaya ${ }^{3}$ \\ 'Department of Radiology, Hendek State Hospital, Sakarya, Turkey \\ ${ }^{2}$ Department of Radiology, Maltepe University School of Medicine, Istanbul, Turkey \\ ${ }^{3}$ Department of Radiology, Bakırkoy Dr. Sadi Konuk Training and Research Hospital, Istanbul, Turkey \\ ${ }^{4}$ Department of Surgery, Hendek State Hospital, Sakarya, Turkey \\ ${ }^{5}$ Department of Radiology, Gebze Medical Park Private Hospital, Kocaeli, Turkey \\ ${ }^{6}$ Department of Radiology, Kagithane State Hospital, Istanbul, Turkey
}

Background: Through Diffusion Weighted Imaging (DWI), information related to early molecular changes, changes in the permeability of cell membranes, and early morphologic and physiologic changes such as cell swelling can be obtained.

Aims: We investigated the correlation between the prognostic factors of breast cancer and apparent diffusion coefficient (ADC) in DWI sequences of malignant lesions.

Study Design: Retrospective cross-sectional study.

Methods: Patients who were referred to our clinic between September 2012 and September 2013, who underwent dynamic breast MRI before or after biopsy and whose biopsy results were determined as malignant, were included in our study. Before the dynamic analysis, DWI sequences were taken. ADC relationship with all prognostic factors was investigated. Pearson correlation test was used to compare the numerical data, while Spearman correlation and Fisher exact tests were used to compare the categorical data. The advanced relationships were evaluated with linear regression analysis and univariate analysis. The efficiency of the parameters was evaluated using ROC analysis. The significance level $(\mathrm{P})$ was accepted as 0.05 .

Results: In total, 41 female patients with an average age of 49.4 years (age interval 21-77) and 44 lesions were included into the study. In the Pearson correlation test, no statistically significant difference was determined between ADC and the patient's age and tumor size. In the Spearman correlation test, a statistically significant difference was determined between nuclear grade $(\mathrm{NG})$ and $\mathrm{ADC}(\mathrm{r}=-0.424, \mathrm{p}=0.04)$; no statistically significant correlation was observed between the other prognostic factors with each other and ADC values. In the linear regression analysis, the relationship of $\mathrm{NG}$ with $\mathrm{ADC}$ was found to be more significant alone than when comparing all parameters (corrected $\mathrm{r} 2=0.196, \mathrm{p}=0.005$ ). Further evaluations between the NG and ADC correlation were carried out with ROC analysis. A statistically significant difference was determined when NG 1 separately was compared with NG 2 and $3(\mathrm{p}=0.03$ ). A statistically significant difference was also determined $(\mathrm{p}=0.05)$ in the comparison of NG 1 with only NG 3. No statistically significant difference was determined when NG 2 separately was compared with NG 1 and NG 3 and when NG 3 separately was compared with NG 1 and $2(\mathrm{p}=0.431, \mathrm{p}=0.097)$,

Conclusion: We found that ADC values obtained by breast DWI showed a higher correlation with the NG of breast cancer, which is an important factor in the patient's treatment. Predictions can be made about NG by analyzing the $\mathrm{ADC}$ values. Additional studies are needed, however, and the $\mathrm{ADC}$ value of the lesion can be used as a prognostic factor proving the aggressiveness.

Keywords: Breast cancer, breast MR, diffusion

This study was presented as a poster presentation at the 12 $2^{\text {th }}$ Balkan Congress of Radiology, 16-19 October 2014, Istanbul, Turkey.

Address for Correspondence: Dr. İnci Kızıldağ Yırgın, Radiology Department, Hendek State Hospital, Sakarya, Turkey

Phone: +905308204689 e-mail: incikizil@hotmail.com

Received: 3 September $2014 \quad$ Accepted: 13 October2015 • DOI: 10.5152/balkanmedj.2016.140555

Available at www.balkanmedicaljournal.org

Kızıldağ Yırgın İ, Arslan G, Öztürk E, Yırgın H, Taşdemir N, Akdoğan Gemici A, et al. Diffusion Weighted MR Imaging of Breast and Correlation of Prognostic

Factors in Breast Cancer. Balkan Med J 2016;33:301-7 
Breast cancer is the most common form of cancer among women worldwide and is also responsible for the most cancerrelated deaths in female cancer patients (1). The number of tumor-related features available to predict the prognosis of patients with breast cancer has grown considerably in recent years. Prognostic factors of breast cancer include histological features (histological subtype, histological grade, nuclear grade (NG), lymphovascular invasion), lymph node status, steroid hormone receptors status, age $(<35$ years) and size of tumor $(>2 \mathrm{~cm})(1,2)$.

Quantitative histopathology can improve the accuracy of prognosis when combined with other objective prognostic criteria, and can also potentially predict response to therapy. Histopathological grade is currently based on the degree of tubule formation, number of mitoses, and NG in routine sections. NG is one of the most important prognostic and predictive markers, and has provided valuable information in many cases $(1,2)$.

Mammography (MG), ultrasound (US) and magnetic resonance imaging (MRI) can help to identify breast lesions more accurately but, nevertheless, remain insufficient for fully characterizing identified lesions. Diffusion-weighted imaging (DWI) is based on the movement of water molecules during the interval of excitation. This motion is affected by the biophysical characteristics of tissue, such as the cell density, membrane integrity and microstructure. Lesions can be characterized according to the diffusion of water in tissue, referred to as the apparent diffusion coefficient (ADC). DWI is sensitive to high cellularity due to proliferation in malignant tumor and the ADC value drops because of the restricted extracellular diffusion. DWI is being used more frequently in patient management due to its ability to evaluate the mobility of water within tissue, and to supply morphological information. Therefore, DWI is a useful tool for tumor detection and characterization as well as for monitoring and predicting treatment response. Although many studies have shown the usefulness of DWI and the ADC value for detecting primary breast lesions (3-5), few have reported correlations between the ADC value and prognostic factors.

To improve the specificity of breast MR analyses, new studies have been carried out by adding DWI to conventional and dynamic sequences. These studies have revealed that DWIs provide additional benefits with respect to dynamic breast MR analysis, by differentiating benign from malignant lesions and identifying breast cancer (6-10).

We investigated the correlation between the prognostic factors of breast cancer and ADC in DWI sequences of malignant lesions by retrospectively evaluating patients who applied to our department.

\section{MATERIALS AND METHODS}

\section{Patients}

Patients referred to our clinic between September 2012 and September 2013 who underwent dynamic breast MRI before or after biopsy, and whose biopsy results were determined as malignant, were included in this study. Ethics Committee approval was obtained from our hospital. Patients were informed of the study procedures and consent was taken before breast MRI. In total, 71 patients were analyzed, but 30 were excluded: 12 because they had received neo-adjuvant chemotherapy and endocrine treatment, 13 had in situ ductal carcinoma on histopathologic evaluation, 2 had medullary carcinoma, 2 had tubular carcinoma, and 1 was excluded for non-diagnosticrelated, technical reasons. Consequently, 41 female patients and 44 lesions were included in the study. The patients' mean age was 49.4 years (range: $21-77$ years).

\section{Imaging methods}

Mammography analysis was performed in the craniocaudal and mediolateraloblique positions as part of a routine analysis protocol. The additional analysis protocols were administered in all patients, with US performed using a linear probe with 5-12 MHz resolution.

Breast MRI was performed with a 1.5 Tesla scanner (Avanto; Siemens, Erlangen, Germany) using standard bilateral breast coils. Spin-echo fat-suppressed T2-weighted axial, precontrast T1-weighted axial and sagittal, enhanced dynamic fat suppression axial, and post-contrast fat-suppression T1weighted axial sequences were performed in all patients.

For dynamic sequences, enhanced images were obtained after the intravenous administration of contrast agents according to the weight $(0.1-0.2 \mathrm{mmol} / \mathrm{kg})$ of the patient. For dynamicenhanced T1-weighted sequences, six images were taken of each section at 30-s intervals. Then, post-contrast fat suppression T1-weighted sequences were obtained. Post-contrast images were excluded from the corresponding pre-contrast images using the subtraction program of a specialized software package.

Before the dynamic analysis, DWI sequences were obtained with the following parameters: repetition time, $1,800 \mathrm{~ms}$; echo time, $81 \mathrm{~ms}$; number of slices, 15; disc-factor, 30; section thickness, $6 \mathrm{~mm}$; Fov read, $380 \mathrm{~mm}$; Fov phase, 100\%; average, 16; concentration, 1; phase direction, anteroposterior; base resolution, 192; plane resolution, $80 \mu \mathrm{m}$; phase partial Fourier, 6/8; PAT mode, Grappa; b values, 0 and $750 \mathrm{sec} / \mathrm{mm}^{2}$; bandwidth, 1,446 Hz/pixel; echo spacing, $0.8 \mathrm{~ms}$; epinephrine factor, 154; gradient mode, fast; and RF pulse, normal. ADC maps were created automatically by using $b$ values of 0 and $750 \mathrm{sec} / \mathrm{mm}^{2}$. Calculations were made based on mean ADC 
maps of the circular sampling region of interest (ROI), with care taken to perform measurements in solid rather than necrotic/cystic areas. The ROIs were $10-100 \mathrm{~mm}^{2}$ in size (ROIs were drawn by a 10 -year experienced radiologist).

\section{Histopathologic analysis}

The histopathological and NG grades of tumors were determined according to the Nottingham grading system (ElstonEllis modification of the Scarff-Bloom-Richardson grading system). Estrogen receptors (ORs) and progesterone receptors (PgRs) were identified using mouse monoclonal antibody (Dako, Glostrup, Denmark); human epidermal growth factor receptor 2 (HER2) score was determined by the Hercep test (Dako), with scores $>3$ considered HER2-positive.

\section{Statistical analysis}

The relationship between ADC and prognostic factors (sentinel lymph node status, tumor size, nuclear grade, lymphovascular invasion, HER2, ER, PgR status, patient age) was investigated. Pearson's correlation was used to compare the numerical data, with Spearman's correlation and Fisher's exact tests used to compare the categorical data. Relationships were also evaluated by linear regression analysis and univariate analysis. The efficiency of the parameters was evaluated using relative operating characteristic (ROC) analysis. A $P$ value $<0.05$ was taken to indicate statistical significance. The statistical analyses were performed using the SPSS for Windows software package (ver. 19.0; SPSS Inc., Chicago, IL, USA).

\section{RESULTS}

In total, $16(36.4 \%)$ and $25(60.9 \%)$ patients were negative and positive, respectively, for sentinel lymph nodes. Lymphovascular invasion was negative in $18(40.9 \%)$ and positive in $26(59.1 \%)$ of the lesions. Eight (18.2\%) lesions were NG 1, $23(52.3 \%)$ were NG 2, and 13 (29.5\%) were NG 3. A total of $32(72.7 \%)$ lesions were HER2-negative, and 12 (27.3\%) were HER2-positive, $9(20.5 \%)$ lesions were ER-negative and 35 (79.5\%) were ER-positive, and 13 (29.5\%) lesions were PgR-negative, and $31(70.5 \%)$ were PgR-positive. A single patient $(2.4 \%)$ patient was $<35$ years of age, and $40(97.6 \%)$ were $>35$ years of age. There were $11(25 \%)$ lesions $<2 \mathrm{~cm}$ in size, and 33 lesions $>2 \mathrm{~cm}$ in size (Figure 1).

There was no significant association between the ADC and patient age or lesion size (Pearson's correlation). There was a significant relationship between NG and the ADC (Spearman's correlation, non-parametric data; $\mathrm{R}=-0.424, \mathrm{p}=0.04$ ). There were no significant correlations between the other prog-

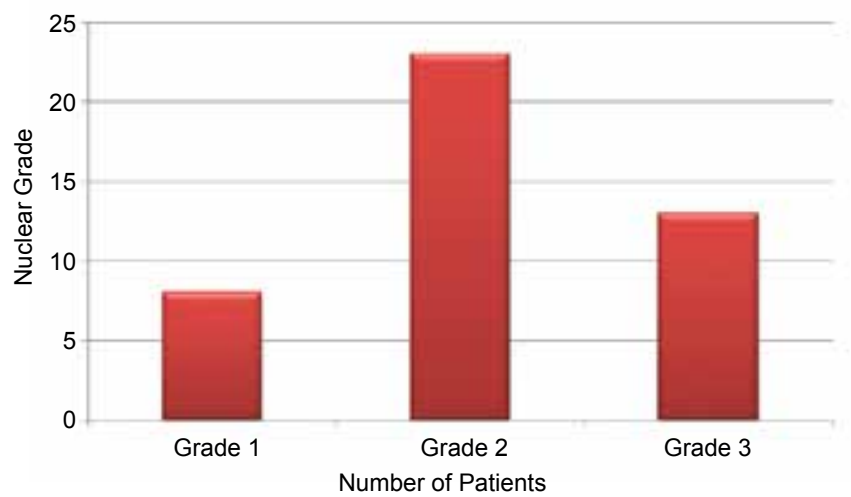

FIG. 1. Nuclear grade classification

TABLE 1. The relationship between the apparent diffusion coefficient (ADC) and nuclear grade $(\mathrm{NG})$ on linear regression analysis

\begin{tabular}{lccc}
\hline $\mathrm{R}$ & $\mathrm{R}$ square & Adjusted R square & Standard error \\
\hline 0.463 & 0.214 & 0.196 & 0.161 \\
\hline
\end{tabular}

TABLE 2. The relationship between ADC and all prognostic factors on linear regression analysis

\begin{tabular}{lccccc}
\hline & $\begin{array}{c}\text { Unstandardized } \\
\text { coefficient } \\
\end{array}$ & \multicolumn{5}{c}{$\begin{array}{c}\text { Standardized } \\
\text { Standard } \\
\text { coefficient }\end{array}$} & $\begin{array}{c}\text { error } \\
\text { Beta }\end{array}$ & $\mathrm{t}$ & Significance \\
\hline Age & 0.003 & 0.002 & -0.230 & -1.504 & 0.142 \\
Size & 0.001 & 0.002 & -0.085 & -0.535 & 0.596 \\
Axillary lymph node & 0.0164 & 0.087 & -0.442 & -1.870 & 0.070 \\
Lymphovascular & 0.193 & 0.093 & 0.535 & 2.072 & 0.046 \\
invasion & & & & & \\
Nuclear grade & 0.120 & 0.040 & -0.462 & -2.999 & 0.005 \\
HER2 & 0.017 & 0.060 & 0.042 & 0.284 & 0.778 \\
OR & 0.031 & 0.081 & 0.071 & 0.385 & 0.703 \\
PgR & -0.129 & 0.71 & -0.332 & -1.821 & 0.077 \\
\hline
\end{tabular}

HER2: human epidermal growth factor receptor 2; OR: estrogen receptor; PgR: progesterone receptor

nostic factors, nor between the other prognostic factors and ADCs. However, there was a significant association between lesion size and lymphovascular invasion $(\mathrm{R}=-0.450, \mathrm{p}=0.02)$.

On linear regression analysis, the relationship between NG and ADC was more significant compared to the relationships between all of the other parameters (corrected $\mathrm{r}^{2}=0.196$, $\mathrm{p}=0.005$; Table 1 and 2). There were no significant correlations between any prognostic factors and ADCs in linear regression analyses for which NGs were not calculated.

As a further step, a univariate analysis was performed in which the effect of NG alone on the ADC was found to be $25 \%$ (corrected $\mathrm{r}^{2}=0.251, \mathrm{p}=0.01$ ). However, the effect of all of the prognostic factors on ADC was calculated as 30\% (corrected $\mathrm{r}^{2}=0.292$ ). 


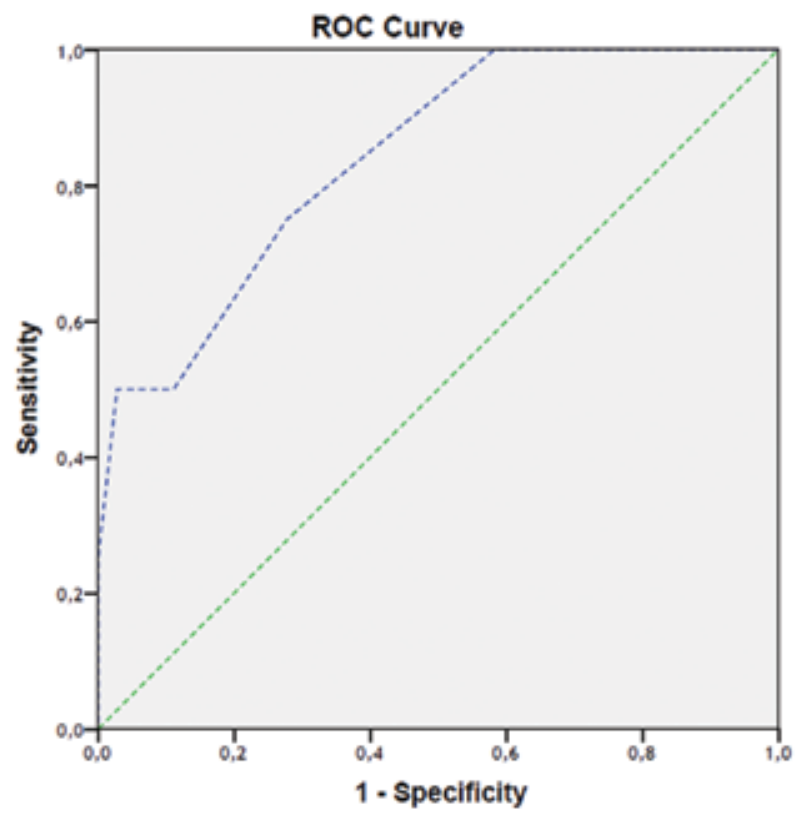

FIG. 2. Relative operating characteristic curve (ROC) in which NG 1 was compared with NGs 2 and 3 (area under the curve $(A \cup C)=0.840$ )

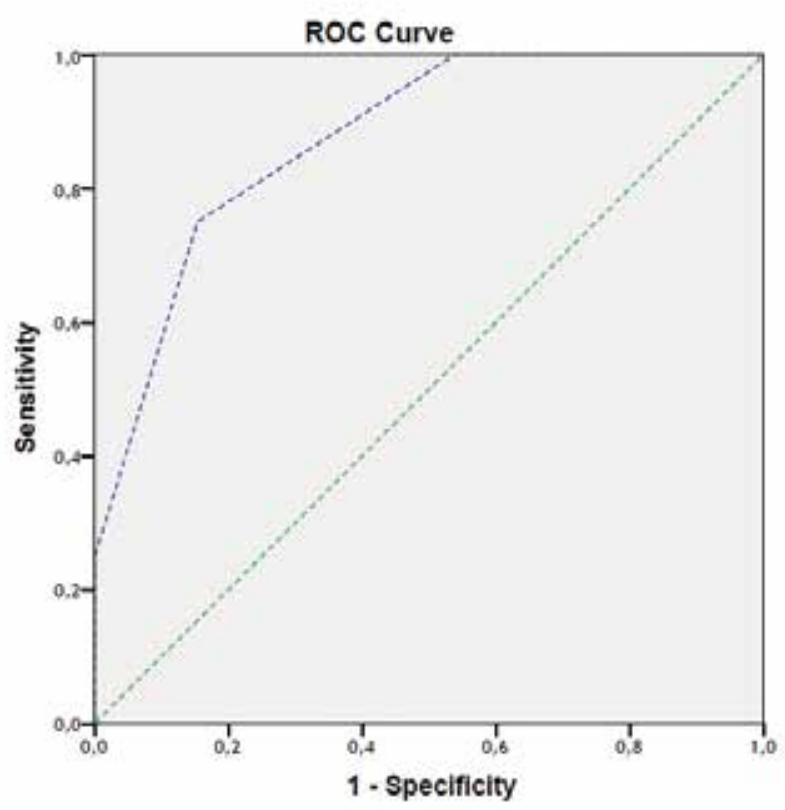

FIG. 3. ROC curve in which NG 1 was only compared with NG 3 $(A \cup C=0.875)$
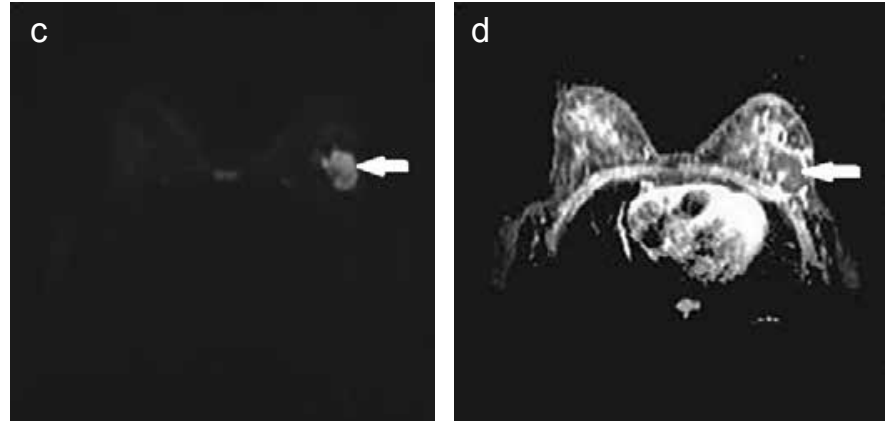

FIG. 4. a-d. A 41-year-old patient diagnosed with left breast invasive ductal carcinoma positive for axillary lymph node, lymphovascular invasion, NG 2, estrogen receptors (ORs), progesterone receptors (PgRs); and negative for human epidermal growth factor receptor 2 (HER2). Enhanced lesion was visualized on fat suppression, T1-weighted post-contrast analysis (a). Heterogeneously enhancing lesion in left breast visualized on subtraction images (b). Hyper-intense lesion on diffusion-weighted images (DWls) (c). The apparent diffusion coefficient (ADC) value was measured as $1.07 \times 10-3 \mathrm{~mm} 2 / \mathrm{sec}$ on ADC maps (d).

Further evaluation of the relationship between NG and ADC was carried out with ROC analysis. There was a significant difference in the degree of association between $\mathrm{ADC}$ and $\mathrm{NG}$ 1 compared to between ADC and NGs 2 and $3(p=0.03)$. A significant difference was also observed in the comparison between NG 1 and NG 3 only, but no significant difference was seen when NG 2 was compared in separate analyses with NGs 1 and 3, nor when NG 3 was separately compared with NGs 1 and $2(p=0.431$ and $p=0.097$, respectively; Figures 2 and 3). Two of our patient's images are provided (Figure 4 and 5).

In ROC curve analysis in which NG 1 was compared with NGs 2 and 3, the ADC cut-off value was calculated as $1.05 \times 10^{-2}$ $\mathrm{mm}^{2} / \mathrm{sec}$ (Table 3). The relationships between all of the prog- nostic factors and ADC, according to the obtained cut-off value, were investigated with Pearson's Chi-square and Fisher's exact test. As with the other tests, no significant correlations were determined, except for between NG and ADC values $(\mathrm{p}=0.020)$.

\section{DISCUSSION}

In recent years, several studies have indicated that DWI can be used to increase the specificity of breast MRI. DWI depends upon the diffusion of water molecules, and on random movement that occurs according to the principles of Brownian 

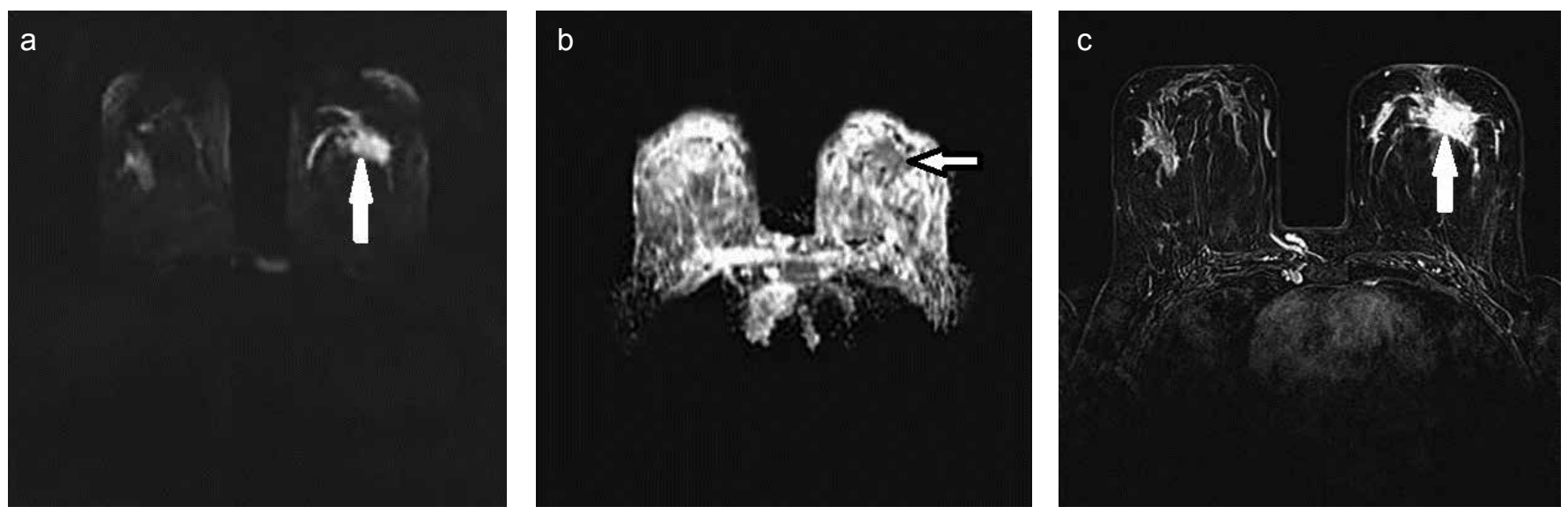

FIG. 5. a-c. A 35-year-old patient diagnosed with left breast invasive ductal carcinoma positive for axillary lymph node, lymphovascular invasion, and NG 3; and negative for ORs, PgRs, and HER2. Hyper-intense lesion on DWI (a), The ADC was measured as 0.95x10-3 mm2/sec on ADC maps (b), Enhanced lesion was visualized on fat suppression, T1-weighted post-contrast analysis (c).

TABLE 3. ADC cut-off value according to the relative operating characteristic curve: comparison of NG 1 with NGs 2 and 3

\begin{tabular}{lcc}
\hline $\begin{array}{l}\text { Positive, if greater } \\
\text { than or equal to: }\end{array}$ & Sensitivity & Specificity \\
\hline-0.300 & 1.000 & 1.000 \\
0.750 & 1.000 & 0.944 \\
0.850 & 1.000 & 0.750 \\
0.950 & 1.000 & 0.583 \\
1.050 & 0.750 & 0.278 \\
1.150 & 0.500 & 0.111 \\
1.250 & 0.500 & 0.028 \\
1.350 & 0.250 & 0.000 \\
1.450 & 0.125 & 0.000 \\
2.500 & 0.000 & 0.000 \\
\hline
\end{tabular}

movement. Through DWI, information related to early molecular changes, changes in the permeability of cell membranes, and early morphological and physiological changes (such as cell swelling and/or cell lysis) can be obtained.

DWI has been used to evaluate cerebrovascular events and numerous types of intracranial lesion. Several studies have shown that DWI is efficient for evaluating the degree of disease and distinguishing benign from malignant breast lesions (8,10-12). By using DWI, quantitative measurements can be obtained in ADC maps of the cellular intensity of the lesion and the diffusion gradient. For example, Kuroki et al. (10) showed that malignant breast lesions had lower ADCs than benign lesions, and invasive ductal carcinoma had lower ADCs compared to in situ ductal carcinoma.

In the present study, individual correlations between all prognostic factors and $\mathrm{ADC}$ values were investigated. In con- trast to many previous studies, average ADC values were not calculated. A significant correlation was only determined between NG and ADC values; as the NG increased, ADC values decreased. No significant correlation was determined between any of the other prognostic factors and ADC values.

Kim et al. (13) found no correlations between any prognostic factor and ADC values and concluded that $A D C$ values were beneficial for identifying malignant lesions, but were not related to the prognosis of the patient. The difference between these authors' study and the present study was that we observed a significant correlation between NG and ADC values.

In a study by Kamitani et al. (14), in which the relationships between prognostic factors and ADC values were compared, higher ADC values were observed in cases that were positive for axillary lymph node, but ADC values were lower in cases that were positive for OR. No correlation was determined between any other factors and ADC values. High ADC values in cases that were positive for axillary lymph node were associated with micro-necrosis and fibrosis inside the lesion. In our study, we did not measure central necrotic/cystic components, and obtained measurements in particular from the solid peripheral region. Moreover, in a study by Razek et al. (15), lower ADC values were observed in cases positive for axillary lymph node, so no clear consensus has yet been reached regarding this association. These authors also investigated correlations between prognostic factors and ADC values and showed that histological grade, tumor size and axillary lymph node metastasis were significantly correlated with ADC; furthermore, as NG and tumor size increased, ADC values decreased. Additionally, ADC values were lower in cases that were positive for axillary lymph node.

Laura et al. (4) investigated the relationships between breast cancer biomarkers and $\mathrm{ADC}$ values and determined that $\mathrm{ADC}$ 
values were lower among OR-positive versus OR-negative cases; furthermore, HER2-negative cases had the highest ADC values. These authors also found a significant relationship between NG and ADC values, similar to the present study. In our study, a significant difference was observed between the degree of association between ADC values and NG 1 compared to between ADC values and NGs 2 and 3, but there was no difference when NG 3 was compared with NGs 1 and 2 in separate analyses. In the study of Laura et al., there was a significant difference between the degree of association between ADC values and NG 3 compared to between ADC values and NGs 1 and 2. This difference may be explained by the fact that the numbers of patients in each NG sub-group were different; nevertheless, both studies found an inverse correlation between NG and ADC values. In a study by Constantini et al. (16), the relationship between ADC values and tumor grade was investigated in 136 patients, with a significant difference determined between the strength of the association between ADC values and NG 1 relative to NGs 2 and 3.

Our study had some limitations. Because it used a retrospective design, a period of specific duration could not be determined between biopsy and MRI. MRI was performed in some patients before biopsy, and in others after biopsy. Furthermore, although the ADC measurement was performed in both solid and peripheral regions of the lesion, differences in $\mathrm{ADC}$ values can also be due to hemorrhages that occur after biopsy. ADC values can vary depending upon the stage of hemorrhage or the width of the cystic/necrosis region. Another limitation of our study was that there were relatively few patients who had undergone adjuvant chemotherapy, and cases of ductal carcinoma in situ were excluded. This could explain why we detected no significant correlation between ORs and ADC values in our study, in general contrast to the results of previous studies.

In conclusion, we found that $\mathrm{ADC}$ values obtained on breast DWI were highly correlated with breast cancer NG; therefore, obtaining ADC values represents an important component of treatment. Predictions can be made regarding NG by analyzing ADC values, although additional studies are needed to validate this. The $\mathrm{ADC}$ of a lesion can also be used as a prognostic factor to assess its aggressiveness.

Ethics Committee Approval: Ethics Committee approval was obtained from Bakırköy Dr. Sadi Konuk Training and Research Hospital (Date: 04.11.2013, Number: 2013/09/05).

Informed Consent: Patients were informed of the study procedures and consent was taken before breast MRI.

Peer-review: Externally peer-reviewed.

Author contributions: Concept - İ.K.Y.; Design - G.A.; Materials - İ.K.Y.; Data Collection and/or Processing - İ.K.Y., G.A., E.K.,
F.Ç.Y., N.T.; Analysis and/or Interpretation - A.A.G., H.Y.; Literature Search - İ.K.Y., H.Y., N.T.; Writing - İ.K.Y., E.Ö.; Critical Reviews - İ.K.Y., E.Ö.

Acknowledgements: Authors would like to thank to Department of Radiology, Bakırköy Dr Sadi Konuk Training and Research Hospital.

Conflict of Interest: No conflict of interest was declared by the authors.

Financial Disclosure: The authors declared that this study has received no financial support.

\section{REFERENCES}

1. Abdalla F, Boder J, Markus R, Hashmi H, Buhmeıda A, Collan Y. Correlation of nuclear morphometry of breast cancer in histological sections with clinicopathological features and prognosis. Anticancer Res 2009;29:1771-6.

2. Donegan WL. Tumor-Related Prognostic Factors for Breast Cancer. CA: A Cancer Journal for Clinicians 1997;47:28-51. [CrossRef]

3. Gouhar GK, El -Hariri MA, Lotfy WE. Malignant breast tumours: Correlation of apparent diffusion coefficient values using diffusion-weighted images and dynamic contrast-enhancement ratio with histologic grading. The Egyptian Journal of Radiology and Nuclear Medicine 2011;42:451-60. [CrossRef]

4. Martincich L, Deantoni V, Bertotto I, Redana S, Kubatzki F, Sarotto I, et al. Correlations between diffusion-weighted imaging and breast cancer biomarkers. Eur Radiol 2012;22:1519-28. [CrossRef]

5. Thomassin Naggara I, De Bazelaire C, Chopier J, Bazot M, Marsault C, Trop I. Diffusion-weighted MR imaging of the breast: Advantages and pitfalls. Eur J Radiol 2012;82:435-6. [CrossRef]

6. Kolb TM, Lichy J, Newhouse JH. Comparison of the performance of screening mammography, physical examination and breast US and evaluation of factors that influence them: an analysis of 27,825 patient evaluations. Radiology 2002;225:165-75. [CrossRef]

7. Segel M, Paulus D, Hortobagyi G. Advanced primary breast cancer: assessment mammography of response to induction chemotherapy. Radiology 1988;169:49-54. [CrossRef]

8. Woodhams R, Matsunaga K, Kan S, Hata H, Ozaki M, Iwabuchi $\mathrm{K}$, et al. ADC mapping of benign and malignant breast tumors, Magn Reson Med Sci 2005;4;35-42. [CrossRef]

9. Rubesova E, Grell AS, De Maertelaer V, Metens T, Chao SL, Lemort M. Quantitative diffusion imaging in breast cancer: a clinical prospective study. J Magn Reson Imaging 2006;24:319-24. [CrossRef]

10. Kuroki Y, Nasu K, Kuroki S, Murakami K, Hayashi T, Sekiguchi $\mathrm{R}$, et al. Diffusion-weighted imaging of breast cancer with the sensitivity encoding technique: analysis of the apparent diffusion coefficient value. Magn Reson Med Sci 2004;3:79-85. [CrossRef] 
11. Lyng H, Haraldseth), Rofstad EK. Measurement of cell density and necrotic fraction in human melanoma xenografts by diffusion weighted magnetic resonance imaging. Magn Reson Med 2000;43:828-3. [CrossRef]

12. Peters NH, Vincken KL, Van Den Bosch MA, Luijten PR, Mali WP, Bartels LW. Quantitative diffusion-weighted imaging for differentiation of benign and malignant breast lesions: the influence of the choice of $\mathrm{b}$ values. J Magn Reson Imaging 2010;31:1100-5. [CrossRef]

13. Kim SH, Cha ES, Kim HS, Kang BJ, Choi JJ, Jung JH, et al. Diffusion-weighted imaging of breast cancer: correlation of the apparent diffusion coefficient value with prognostic factors. $J$ Magn Reson Imaging 2009;30:615-20. [CrossRef]
14. Kamitani T, Matsuo Y, Yabuuchi H, Fujita N, Nagao M, Jinnouchi M, et al. Correlations between apparent diffusion coefficient values and prognostic factors of breast cancer. Magn Reson Med Sci 2013;12:193-9. [CrossRef]

15. Razek AA, Gaballa G, Denewer A, Nada N. Invasive ductal carcinoma: correlation of apparent diffusion coefficient value with pathological prognostic factors. NMR Biomed 2010;23:619-23. [CrossRef]

16. Constantini M, Belli P, Rinaldi P, Bufi E, Giardina G, Franceschini $G$, et al. Diffusion-weighted imaging in breast cancer: relationship between apparent diffusion coefficient and tumour aggressiveness. Clin Radiol 2010;65:1005-12. [CrossRef] 